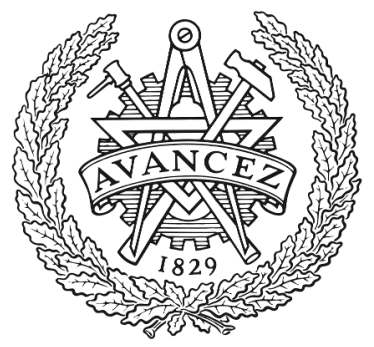

CHALMERS

UNIVERSITY OF TECHNOLOGY

\title{
Homogeneous differential magnetic assay
}

Downloaded from: https://research.chalmers.se, 2023-04-26 15:26 UTC

Citation for the original published paper (version of record):

Sepehri, S., Zardán Gómez De La Torre, T., Schneiderman, J. et al (2019). Homogeneous differential magnetic assay. ACS Sensors, 4(9): 2381-2388. http://dx.doi.org/10.1021/acssensors.9b00969

N.B. When citing this work, cite the original published paper. 


\title{
Homogeneous differential magnetic assay
}

\author{
Sobhan Sepehri ${ }^{*}$, Teresa Zardán Gómez de la Torre‡, Justin F. Schneiderman†§, Jakob Blomgren", \\ Aldo JesorkaT, Christer Johansson", Mats Nilsson', Jan Albert"-, Maria Strømme‡, Dag Winkler†, \\ and Alexei Kalaboukhov ${ }^{\dagger}$
}

\author{
† Department of Microtechnology and Nanoscience - MC2, Chalmers University of Technology, SE-412 96 Göteborg, \\ Sweden. \\ ‡ Department of Engineering Sciences, Uppsala University, The Ångström Laboratory, Box 534, SE-751 21 Uppsala, \\ Sweden. \\ $\S$ MedTech West and the Institute of Neuroscience and Physiology, University of Gothenburg, SE-40530 Göteborg, \\ Sweden. \\ " RISE - Research Institute of Sweden, SE-411 33 Göteborg, Sweden. \\ ॠ Department of Chemistry and Chemical Engineering, Chalmers University of Technology, SE-412 96 Göteborg, Swe- \\ den. \\ ${ }^{\perp}$ Science for Life Laboratory, Department of Biochemistry and Biophysics, Stockholm University, Box 1031, \\ SE-171 21 Solna, Sweden. \\ $\square$ Department of Clinical Microbiology, Karolinska University Hospital, Stockholm, Sweden. \\ - Department of Microbiology, Tumor and Cell Biology, Karolinska Institute, SE- 17176 Stockholm, Sweden. \\ KEYWORDS: homogenous differential magnetic assay, magnetic nanoparticle, volumetric detection, rolling circle amplifi- \\ cation, Brownian relaxation, binding kinetics.
}

\begin{abstract}
Assays are widely used for detection of various targets, including pathogens, drugs, toxins etc. Homogeneous assays are promising for the realization of point-of-care diagnostics as they do not require separation, immobilization, or washing steps. For low concentrations of target molecules, the speed and sensitivity of homogeneous assays have hitherto been limited by slow binding kinetics, time-consuming amplification steps, and the presence of a high background signal. Here, we present a homogeneous differential magnetic assay that utilizes differential magnetic readout that eliminates previous limitations of homogeneous assays. The assay uses a gradiometer sensor configuration combined with precise microfluidic sample handling. This enables simultaneous, differential, measurement of a positive test sample containing synthesized Vibrio Cholera target and a negative control sample, which reduces background signal and increases the read-out speed. Very low concentrations of targets down to fM levels are thus detectable without any additional amplification of number of targets. Our homogeneous differential magnetic assay method opens new possibilities for rapid and highly sensitive diagnostics at the point-of-care.
\end{abstract}

Assays are analytical tools for qualitative and quantitative detection of a target analyte. The target analytes could be of biological (e.g. antibodies, DNA/RNA molecules, proteins, enzymes, etc ${ }^{1}$ ) or non-biological nature (e.g. ions, dissolved gases, drugs and toxins ${ }^{2}$ ). Diverseness, high sensitivity and specificity of assays are crucial in pharmaceutical analysis, drug monitoring, diagnosis of diseases, clinical pharmacokinetic and bioequivalence studies, environmental monitoring and pollutant detection. Assays are immensely powerful in clinical analysis of viruses and various diseases, where rapid and timely diagnosis is crucial for public health.

Standard assays are often laborious, time consuming, and require a specialized laboratory with trained personnel. There is an increased need for rapid, portable, and userfriendly assays that can perform point-of-care (POC) diagnostics at the home, emergency rooms, developing countries and resource-limited environment ${ }^{3}$. Homogenous assays are advantageous for POC diagnostics as they do not require washing steps, separation, and immobilization ${ }^{4}$.
They use the volume of the solution for sample preparation and signal generation and three-dimensional diffusion results in a more rapid binding kinetics, reducing the total turn-around time ${ }^{5}$. Combined with microfluidic-based devices, homogeneous assays offer an opportunity for sensitive and cost-effective POC diagnostics ${ }^{1}$.

In homogeneous assays, the detection signal can be generated from specific binding reaction between the targets and the labels. The labels are usually mixed in solution with the test sample and the signal is generated in the entire mixed volume. In practice, the concentration of labels should be comparable with the concentration of targets in order to have a detectable signal. However, the time required to reach an equilibrium in binding kinetics strongly depends on the concentrations of target and labels. Therefore, measurement of low target concentration is a challenge. Various amplification methods, including isothermal enzymatic schemes ${ }^{6}$ and the polymerase chain reaction $(\mathrm{PCR})^{7}$ have been applied to increase the readout signal and reach sub-fM detection levels in homogeneous assays ${ }^{8,9}$. 

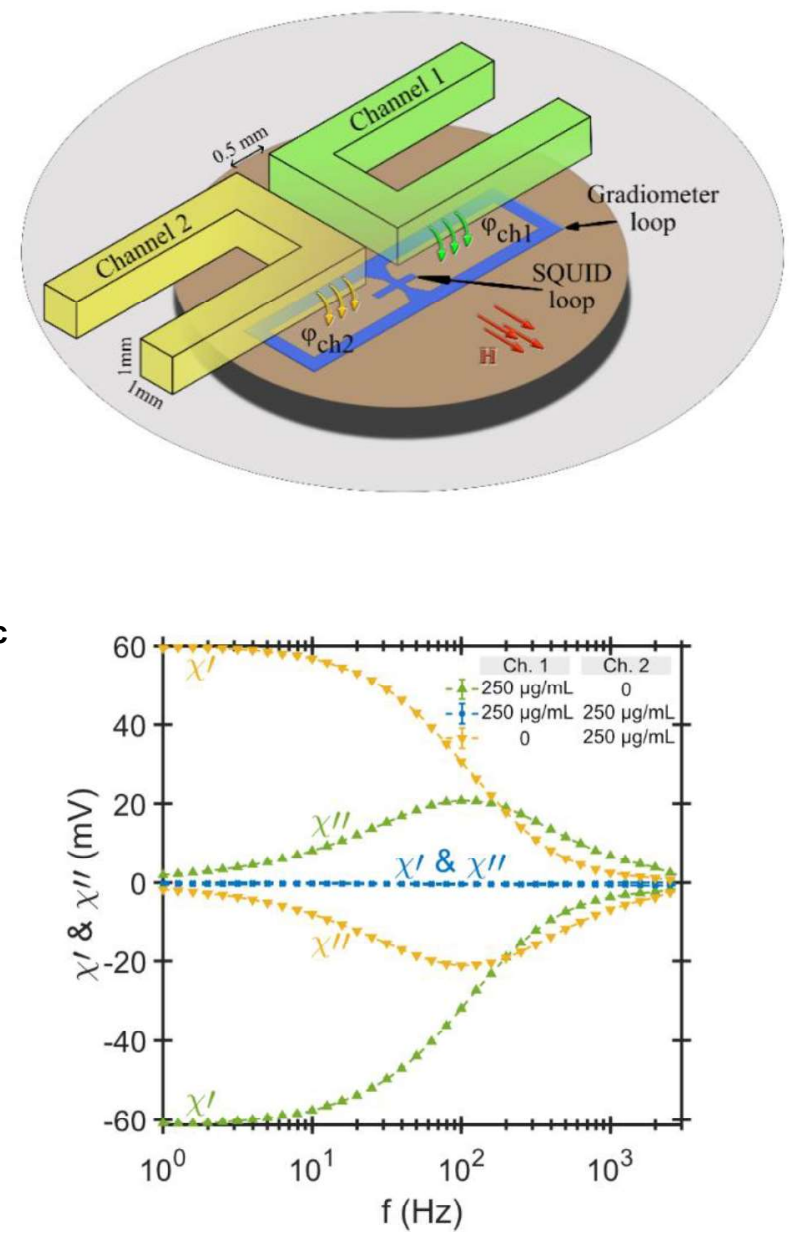

b

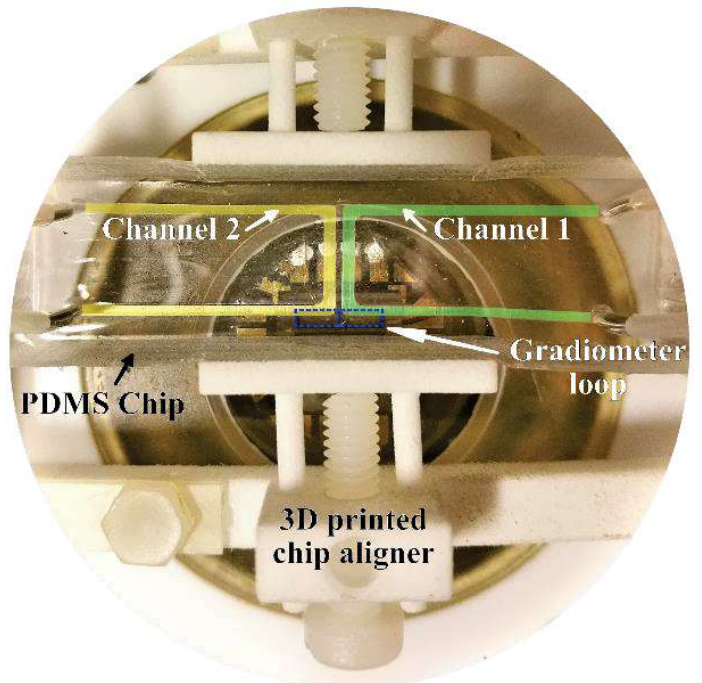

d

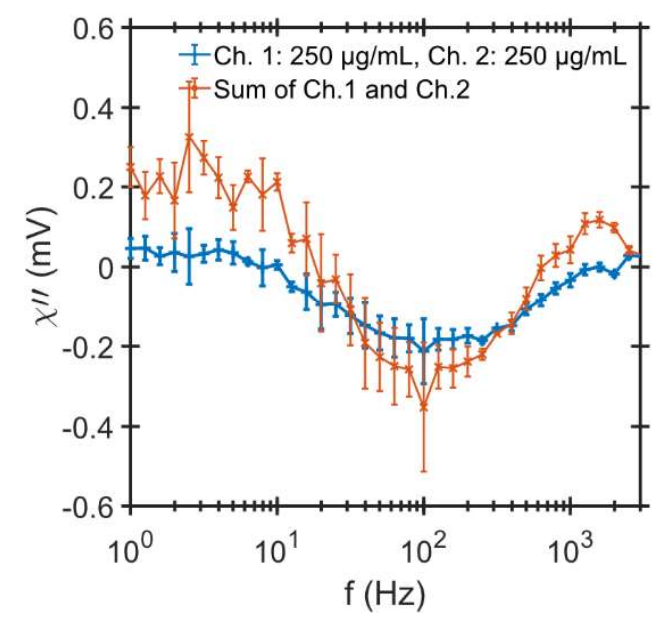

Figure 1 | Differential magnetic assay using the microfluidic device. (a) Schematic illustration of the readout configuration. Two microfluidic channels are integrated into a PDMS chip and is aligned above a 1st order planar gradiometer SQUID sensor. An ac magnetic excitation field $(\mathbf{H})$ is applied to the channels, parallel to the sensor chip so that the SQUID is insensitive to it. In this configuration, the magnetic fluxes produced by the MNPs in the two channels are subtracted. (b) A top view photograph of the microfluidic chip aligned above the SQUID sensor; the channels are highlighted in yellow and green. (c) Real and imaginary components of the ac magnetic susceptibility from $250 \mu \mathrm{g} / \mathrm{mL}$ MNP samples filling: only channel 1 (green triangles), only channel 2 (yellow triangles), and both channels (blue circles) versus the frequency of the magnetic excitation field. Note that the magnetic ac susceptibility signals from channel 1 is opposite in sign compared to that of channel 2 due to the differential measurement setup. (d) The measured imaginary component of ac magnetic susceptibility when both channels are filled. For comparison, the sum of the signals from the individual channels shown in Fig. 1c is also plotted. This magnetic ac susceptibility signal is considered the zero signal of the system and is 100 times lower in magnitude compared to the individual signals in channel 1 or 2 . The summation of the magnetic ac susceptibility signals from the individual channels results in the same response as obtained from the measurement. The ac susceptibility values are expressed as a voltage read out from the SQUID electronics.

The drawbacks of nucleic acid amplification are the risk of cross-contamination leading to false positive detection, and relatively high cost. Reducing assay complexity and total processing time while preserving high sensitivity and specificity is still one of the major challenges in POC diagnostics.

Here, we present a differential magnetic assay based on microfluidic sample handling that circumvents the main limitations of the homogeneous assay. Magnetic assays use bio-functionalized magnetic nanoparticles (MNPs) as labels ${ }^{10}$. Bio-functionalized MNPs can efficiently and selectively bind to biological targets both as labels or as a substrate for binding ${ }^{11}$. Biological samples generally contain no magnetic materials and thus no magnetic background that may obscure highly sensitive magnetic measurements ${ }^{12}$. Bio-functionalized MNPs have been successfully utilized as contrast agents in magnetic resonance imaging, disease therapy, biochemical separation, as well as for bioanalysis and immunoassays ${ }^{13,14}$. Target recognition in our magnetic assay exploits the specific binding reaction between colloidal MNPs and the targets to induce an increase in the hydrodynamic volume of the MNPs. This, in 
turn, changes the characteristic relaxation time of the MNPs and can be measured using magnetic ac susceptibility ${ }^{15}$. In our assay, we use a gradiometer coupled magnetic sensor configuration to simultaneously measure the magnetic ac susceptibility from positive test and negative control samples. The differential readout enables subtraction of the excess unbound MNP labels in the test sample and allows measurements of fM target concentrations without altering the rate of binding kinetics. This drastically shortens the assay time while preserving sensitivity and specificity, that are comparable with laboratory-based assays.

\section{Experimental method}

Our approach utilizes two identical microfluidic channels fabricated in a single polydimethylsiloxane (PDMS) device. The channels may be individually filled with magnetic fluid samples. The magnetic samples are excited by an ac magnetic field and their magnetic ac susceptibility is measured using a planar gradiometer sensor. The frequency dependent magnetic ac susceptibility provides information about the effective relaxation time which is dependent on the particle size distribution of the MNP colloidal suspension. Each microfluidic channel is aligned parallel to the baseline of the gradiometer along the pickup loops, as illustrated in Fig. 1a. In this geometry, the screening currents produced by magnetic flux from the magnetic fluids in each individual channel are subtracted in the middle line of the gradiometer where a high-temperature superconducting quantum interference device (SQUID) sensor is situated. Fig. 1b shows an image of the aligned PDMS device on the gradiometer using a 3D printed $x-y$ alignment stage. Full details and description of the materials, methods and equipment including the detection of the target molecule, i.e. synthesized Vibrio Cholera, and the amplification process are presented in the Supporting Information SI. 1.

\section{Differential ac susceptibility measurements}

First, we demonstrate the ability of the differential readout to cancel signals from two identical magnetic samples filled in both channels and measured at rest. The magnetic samples are MNPs with a median particle diameter of $100 \mathrm{~nm}$ and no surface functionality. Fig. 1c shows the magnetic ac susceptibility responses from the MNPs with concentration of $250 \mu \mathrm{g} / \mathrm{mL}$. The ac susceptibility is expressed as a voltage read out from the SQUID electronics. Since the actual susceptibility is only a calibration factor that converts the voltage to a sample magnetic moment (and ac susceptibility by dividing magnetic moment with field amplitude and sample volume) the voltage values are used as a measurand of the ac susceptibility. When both channels are filled with the same sample, the response signal is approximately zero and matches well with the manually added signals from the individually-filled channels. Fig. $1 \mathrm{~d}$ shows the differential measurement and the manually added ac susceptibility signals from individual channels and indicates lower errors in the differential measurement. The similarity of the two ac susceptibility responses shows that the measured ac susceptibility signal is a linear superposition of the positive and negative signals from channel 1 and 2 , respectively. When measuring two different samples filling channels 1 and 2, the expected result is proportional to the relative difference in the ac susceptibility of the two samples which could, for example, be a result of a relative difference in the particle size distributions. The differential signal is about $0.5 \%$ of the amplitude of the signals from the individual channels. The standard

a

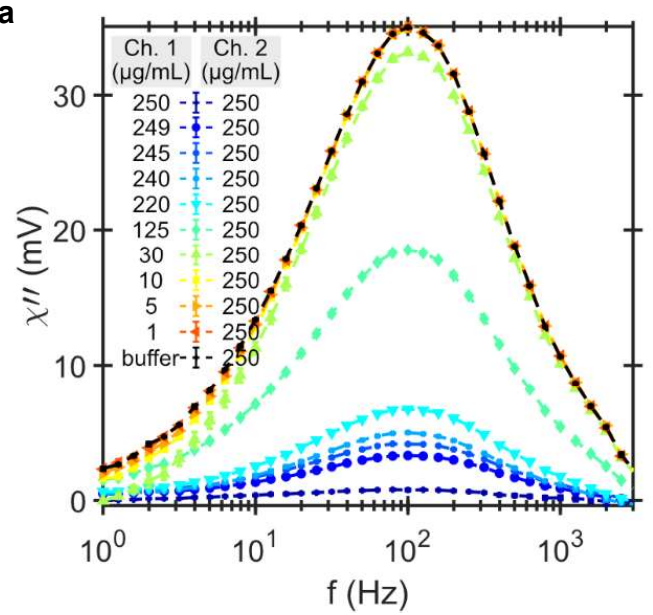

b

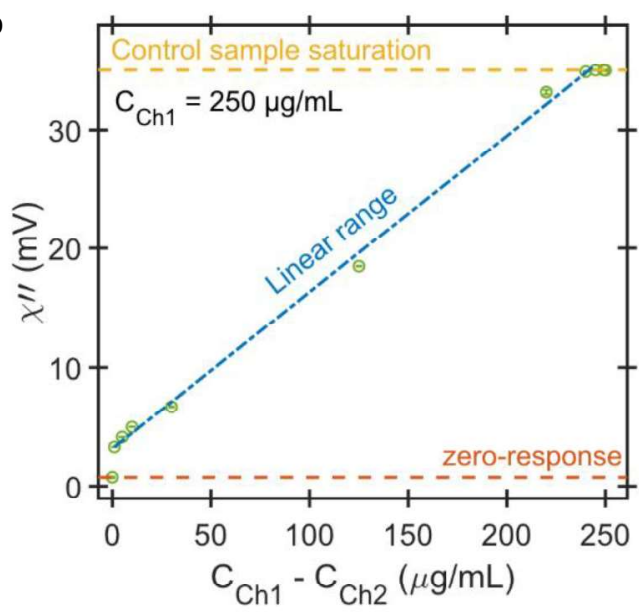

Figure 2 | Calibration of magnetic sensitivity for the differential magnetic readout. (a) Differential measurements of the imaginary component of the magnetic ac susceptibility for several test samples with different concentrations of MNPs ranging from $1 \mu \mathrm{g} / \mathrm{mL}$ to $250 \mu \mathrm{g} / \mathrm{mL}$ in channel 1 with a reference sample in channel 2 containing $250 \mu \mathrm{g} / \mathrm{mL}$ MNPs. Error bars are standard deviation. (b) The amplitude of the imaginary component of the differential ac susceptibility peak at $100 \mathrm{~Hz}$ versus the difference in the concentration of magnetic material in the two channels. When the difference in the two concentrations is large (i.e., $\boldsymbol{C}_{\boldsymbol{C h} \mathbf{1}}-\boldsymbol{C}_{\boldsymbol{C h} \mathbf{2}}$ approaches $\boldsymbol{C}_{\boldsymbol{C h} 2}$ ), the background magnetic signal makes it difficult to distinguish small differences in the test sample and the imaginary susceptibility saturates to that of the control sample signal amplitude. The blue line indicates the linear range of the $\boldsymbol{C}_{\boldsymbol{C h} \mathbf{1}}-\boldsymbol{C}_{\boldsymbol{C h} \mathbf{2}}$ (excluding the zero-response and the saturation values) and has a regression co-efficient of 0.99 . 
deviation in the differentially measured signal is also much smaller compared with manually added signal from individual channels.

In the next step, we calibrate the magnetic sensitivity of our readout system by measuring different concentrations of MNPs in one channel, while a control sample with a fixed MNP concentration is present in the other. The concentration of the control sample is $250 \mu \mathrm{g} / \mathrm{mL}$, while test samples contain MNPs with concentrations ranging from 1 to 250 $\mu \mathrm{g} / \mathrm{mL}$. Fig. 2a shows the imaginary part of the differential ac susceptibility as a function of frequency for different concentration of MNPs in the test samples. Starting with a test sample concentration of $250 \mu \mathrm{g} / \mathrm{mL}$, we obtain zero response $\left(C_{\text {control }}-C_{\text {test }}=0\right)$. By decreasing the MNP concentration of the test sample, the imaginary part of the differential ac susceptibility increases as expected. Fig. 2b shows the imaginary part of the differential ac susceptibility at the peak amplitude frequency of $100 \mathrm{~Hz}$ as a function of the difference in the MNPs concentration in the two channels. When the concentration of the test sample becomes significantly small as compared to that of the control sample, i.e. when $C_{\text {control }}-C_{\text {test }} \approx C_{\text {control }}$, the differential ac susceptibility signal approaches that of the control sample (see Fig. 2a). When the particle concentrations in the two channels are approximately equal, even if the particle concentration in each individual channel is high, the differential susceptibility measurement gives a low signal, which corresponds to the minute difference between the samples. Therefore, the differential readout is highly sensitive to small differences between magnetic samples. This is crucial for the realization of homogeneous magnetic assays that aim for detecting low concentrations of target molecules. Using a calibration curve (c.f., Fig. 2b), we can precisely calculate the difference between the concentrations of MNPs in the two samples, assuming they have exactly the same particle size distribution. For samples with different particle size distributions, the full frequency response should be measured in order to obtain information about the difference in size distributions between the two samples.

\section{Detection of Vibrio Cholera DNA using differential ac susceptibility readout}

In our magnetic assay, we measure changes in magnetic ac susceptibility of the MNPs due to specific binding reactions with target molecules. The difference in the ac susceptibility signal could be either a shift in frequency, due to a change in the hydrodynamic volume of the MNPs, or a reduction in signal amplitudes, so-called turn-off detection ${ }^{16}$. A change in the hydrodynamic volume of MNPs due to binding reactions with analyte molecules is rather small and difficult to detect due to the broad particle size distribution of the MNPs and small increase in particle size ${ }^{15}$. In the turnoff detection method, the MNPs that are specifically bound with the target analyte are taken out of the main particle size distribution due to a large increase in the hydrodynamic volume of the particle-target complex. The bound MNPs then shift out from the measuring frequency window if they, together with the target to which they are bound, are much larger compared to the free unbound MNPs, and the remaining signal is only due to free/unbound MNPs. Therefore, the extinction of the peak amplitude of the imaginary part of the ac susceptibility is proportional to the number of target analyte molecules. When the concentration of the target analyte is much smaller than the MNP concentration, the extinction signal is too small to measure. To detect very low concentrations of analyte, one should match the concentration of MNPs with the analyte concentration in order to have a significant fraction of the MNPs bound to the target. This is a significant drawback of turn-off detection as the binding kinetics become slow at low concentrations, and the overall time of the assay significantly increases in order to keep sensitivity high. The differential ac susceptibility method removes the magnetic background signal that arises from the excess unbound MNPs. Therefore, a low concentration of magnetic probes is not required in order to measure low concentrations of analytes.

To demonstrate this, we perform differential measurements of positive test samples containing different concentrations of rolling circle products (RCPs) of synthetic V. Cholera DNA targets and a negative control (NC) sample. The RCPs are a result of volume amplification using rolling circle amplification (RCA) and each individual RCP in the test sample corresponds to one single $\mathrm{V}$. Cholera target. The NC sample is prepared by mixing the MNPs with a hybridization buffer; it therefore does not contain any RCPs. We emphasize that all of the positive test samples and the NC sample contain the same amount of MNPs (particle concentration of $250 \mu \mathrm{g} / \mathrm{mL}$ in $3.6 \mu \mathrm{L}$ sample volume). The zeroresponse signal, in this case, is the measured signal when both channels are filled with the NC. Fig. 3a shows the imaginary part of differential ac susceptibility as a function of frequency for the NC sample and a series of positive test samples with different concentrations of RCPs. Due to the surface functionalization of the MNPs, the monomodal size distribution of the MNP turns into a bimodal one. Therefore, a bimodal Brownian relaxation model consisting of a superposition of two Cole-Cole models (See Supporting Information SI. 3) is fitted to the data, solid lines in Fig 3a. The fitting parameters are listed in Supporting Table 1. The peak amplitudes increase when the concentration of RCPs increases from 0 to $90.4 \mathrm{pM}$. The increase of the peak amplitude in our differential readout is due solely to the change of the MNP size distribution in the positive test sample. The hydrodynamic volume of the MNP-RCP complex is larger than that of the unbound MNPs. Therefore, the Brownian relaxation frequency of the MNP-RCP complexes is shifted to lower frequencies in the positive test sample. At equilibrium, the fraction of MNP probes bound to RCPs to the total number of MNPs is defined as fraction bound, $f b_{e q}$, which is related to the concentration of the RCPs and the equilibrium dissociation constant, $K_{D}$, and is proportional to the sensing signal: $f b_{e q}\left(C_{R C P}\right)=\frac{C_{R C P}}{C_{R C P}+K_{D}} \propto \chi^{\prime \prime}\left(C_{R C P}\right) / \chi_{\text {Max }}^{\prime \prime}$, where $\chi_{M a x}^{\prime \prime}$ is the signal amplitude from the NC sample in channel 1 (see Supporting Information). Assuming that the magnetic moment of individual MNP does not depend on its volume, the amplitude of the imaginary component of the ac susceptibility is proportional to the concentration of the 
a

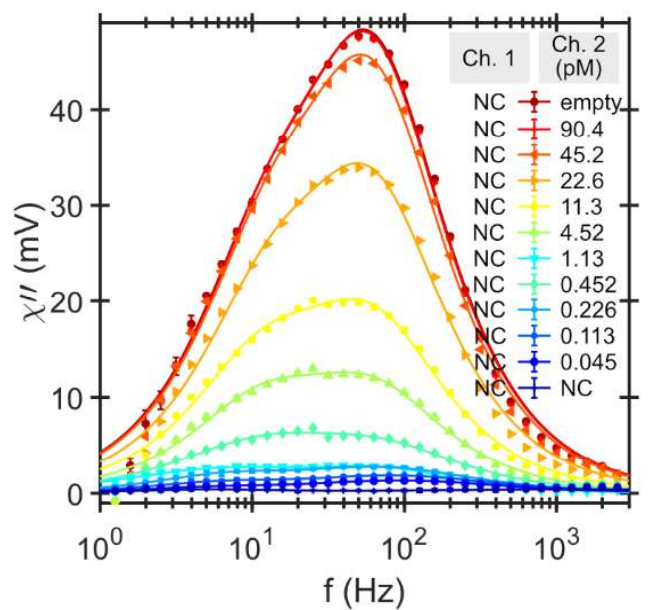

c
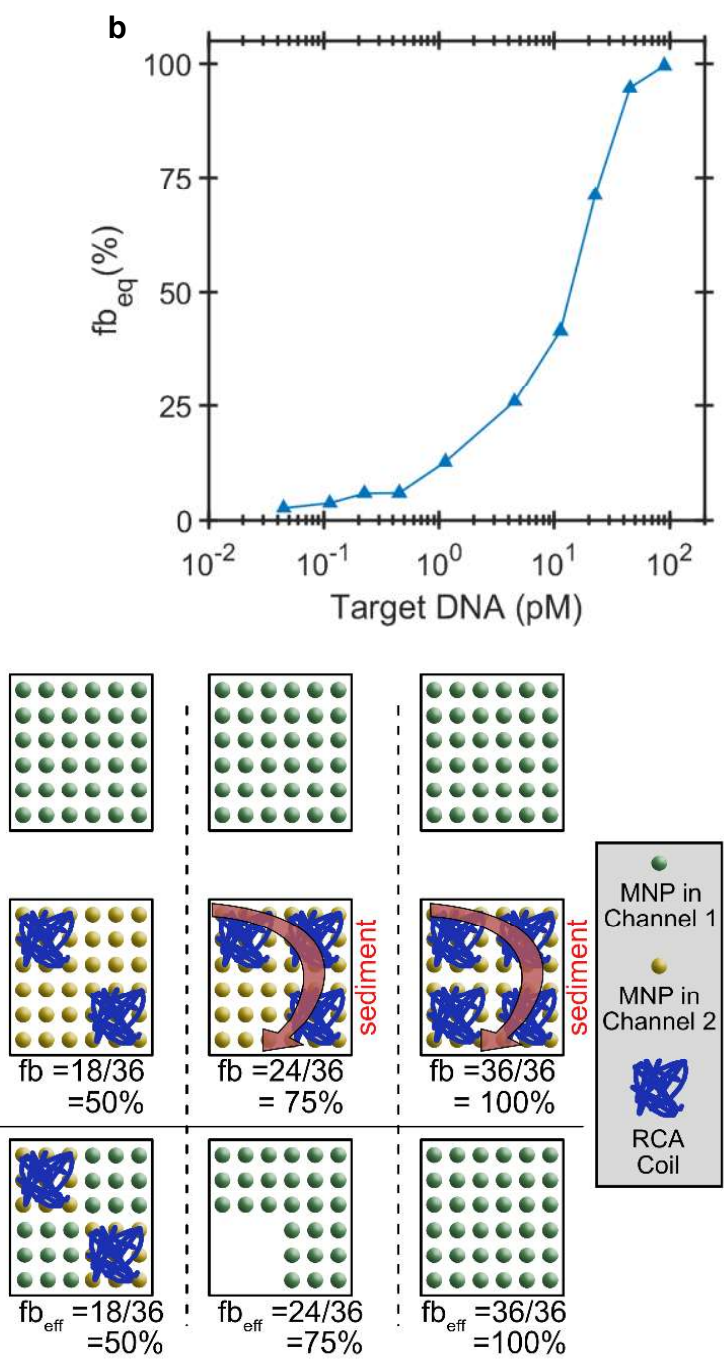

Figure 3 | Quantification of the DNA target concentration using differential ac susceptibility measurements. (a) Imaginary component of differential ac susceptibility versus the frequency for positive test samples containing different concentrations of RCPs ranging from $45 \mathrm{fM}$ to $90 \mathrm{pM}$ (one RCP corresponds to one DNA target). The NC sample is filled into channel 1 and the positive test samples are filled into channel 2. The solid lines are fits to the data with a bimodal Brownian relaxation model consisting of a superposition of two Cole-Cole models for the free MNPs and the MNP-RCP complexes. (b) The equilibrium fraction bound measured as the normalized imaginary signal of the ac susceptibility at $64 \mathrm{~Hz}$ versus the concentration of the V. Cholera DNA targets (RCPs) in the $3.6 \mu \mathrm{L}$ sample volume. The LOD is $45 \mathrm{fM}$ and the working range spans more than 3 orders of magnitude in target concentration. (c) A cartoon illustration of the dynamic equilibrium in each individual channel and what is measured by the sensor in the differential readout for varying binding fractions. For $C_{R C P} \leq K_{D}$, the effective fraction bound is always $50 \%$ (i.e., for each MNP that is bound in channel 1, there is a corresponding free MNP in channel 2). For RCP concentrations above the equilibrium dissociation constant, the RCPs and MNPs agglomerate and sediment (red arrows), which takes the bound MNPs in the positive test sample out of the measurement window. In this case, the sensor signal is the difference between that of the MNPs in the NC sample (channel 1) and the remaining (unbound) MNPs in the test sample (channel 2). This results in an effective binding fraction of more than $50 \%$.

MNPs. The fraction bound measured at the characteristic relaxation frequency of unbound MNPs at $64.5 \mathrm{~Hz}$ therefore, depends on the concentration of the RCPs in the positive test sample and follows a logistic function behaviour ${ }^{17,18}$, as shown in the calibration plot in Fig. 3b. Fig. 3c is a cartoon representation of the equilibrium dynamics in the NC sample (channel 1) and the positive test sample (channel 2), and the difference between the two, which is measured in the differential readout. In the case of turn-off detection method, when the number of MNPs significantly exceeds the number of RCPs, $C_{M N P} \gg C_{R C P}$, it is difficult to detect low concentrations of RCPs as the fraction of bound MNPs is close to zero. To detect such low concentrations of RCPs, a measurable fraction must be bound, which typically means that the concentration of the MNP probes should be lowered to a level where it is comparable with the concentration of 
the RCPs ${ }^{18}$. This, however, results in a very low signal from the probes and requires a longer time to reach equilibrium. Our differential readout eliminates the excess background signal from the MNPs in the positive test sample without changing the concentration of the MNP probes. As a result, the sensor measures only the MNP-RCP complexes and the MNP probes consumed by RCPs, which corresponds to the effective fraction bound of $50 \%$. This can be seen as an effective reduction of concentration of MNPs without changing the binding kinetics. A 50\% fraction bound holds for all concentrations of RCPs where $C_{R C A}<K_{D}$ and the number of MNP probes can be assumed to exceed the number of RCPs. At higher concentrations of RCPs, where $C_{R C A} \geq K_{D}$, the fraction bound becomes larger than 50\% until all MNP probes in the positive test sample are bound (see Supporting Table 1 for an estimation of the available number of MNPs per RCP at different concentrations of RCPs). At $C_{R C P}=K_{D}$, the fraction bound is $50 \%$. At this point, the sensor signal is $50 \%$ of its maximum which means that half of the MNP probes are hybridized to the RCPs. Using the calibration plot in Supporting Fig. 2, we estimate the dissociation constant of our assay to be between 10 and 20 pM. Since the imaginary ac susceptibility at $64.5 \mathrm{~Hz}$ in this range is a linear function of RCP concentration, we estimate the equilibrium dissociation constant to be $K_{D}=16.5 \mathrm{pM}$. Having the equilibrium dissociation constant and the total number of available MNP probes, we can also estimate the average number of MNPs in the MNP-RCP complex to be: $g_{M N P}=$ $\frac{C_{M N P}}{2 K_{D}} \approx 7.5$. This is 2-3 times more MNPs per RCP compared to previously reported estimations ${ }^{19,20}$. This increased number of MNPs per RCP further increases the sensitivity of our assay.

\section{Discussion}

Having no washing step in a homogenous assay greatly simplifies its implementation for POC diagnostics. In such assays, the detection signal, which is a result of the binding interaction between the analyte and the probe, is not separated from the background signal ${ }^{5}$. In homogenous magnetic assays ${ }^{21}$, the background signal comes from the excess magnetic probes (in our case the unbound MNPs) and limits the sensitivity of the assay. Practically, one can control the concentration of the labels to be comparable with the concentration of the targets in order to always ensure that a measurable fraction of the analytes is bound to the detection probes. However, at low concentrations, the binding kinetics becomes slower and increases the necessary time to reach equilibrium. Therefore, measurements of low target concentrations are challenging in magnetic homogeneous assays. Our differential magnetic readout eliminates the background signal without modifying the binding kinetics. It is analogous to a washing step where the excess number of unbound labels is removed from the positive test sample. This allows us to keep a high label to target molecules ratio without affecting the binding kinetics. The kinetics in our assay is thus determined by the high concentration of the labels, and equilibrium is reached after the same hybridization time for any concentration of the targets. The total turn-around time of the homogenous differential magnetic assay is 60 minutes. We have reported that the turn-around time for the assay in the turn-off detection approach is 115 minutes ${ }^{20}$.

The simultaneous measurement of both positive test and negative control samples further reduces the measurement time. Real-time comparison enables accounting for possible sources of variation in the signals, which are hard to control, including unspecific binding to the microfluidic walls, sedimentation, and clustering of the MNPs. This results in a robust measurement of weak positive signals and increases the precision of the measurements as the stability of the colloidal samples at such small volumes suffer from time variation $^{22}$. Our differential magnetic readout has enabled us to reach a fM level of sensitivity without additional amplification of the number of targets. We should emphasize that the RCA technique is only used here for volume amplification and not for increasing the number of target copies.

A typical sensitivity for previously reported magnetic assays is about 1-10 $\mathrm{pM}^{19,23-31}$, see Supporting Table 2. Using very low concentration of MNP probes, i.e., $5 \mu \mathrm{g} / \mathrm{mL}$, Tian et al. ${ }^{31}$ has reported $780 \mathrm{fM}$ LOD which is 15 times better than the LOD reported for $100 \mu \mathrm{g} / \mathrm{mL}$ of magnetic probes ${ }^{30}$. Such an improvement in the LOD is an inherent result of decreasing magnetic probe concentration which, in turn, increased the binding fraction. Despite our relatively high concentration of MNPs (250 $\mu \mathrm{g} / \mathrm{mL})$, our differential signal corresponds to an effective $50 \%$ binding fraction, which results in an experimental LOD of $45 \mathrm{fM}$. This clearly highlights the importance of differential readout and background elimination in our assay. A significant benefit is that the necessary hybridization time required to reach equilibrium does not increase with decreasing RCP concentrations.

The experimental LOD ( $45 \mathrm{fM}$ ) that we have reached in our assay is still two orders of magnitude above the noise level of our magnetic sensor. The linear dynamic range for our assay is 3 orders of magnitude. Theoretically, we could reach an LOD as low as $0.4 \mathrm{fM}$ at a signal-to-noise ratio of 10 and extend the dynamic range to five orders of magnitude, see Supporting Fig. 2. Practically, the LOD is greatly affected by the colloidal stability and fluctuations in the concentration of the nanoparticle system in small volumes. This is a generic problem that did not allow us to measure lower concentrations of targets, as we observe signal variations that exceed the noise level of our sensor. This implies that it is not necessary to utilize sensors with extreme noise performance (such as the SQUID we used) in order to reach fM levels of sensitivity. Technically, one can take advantage of the symmetries in many other magnetic sensors to subtract the magnetic background. Magnetic sensors including flux gates $^{32,33}$, induction coils ${ }^{15,29}$, and giant magnetoresistive sensors ${ }^{34}$ are examples of sensors that have reasonably high magnetic field sensitivity and can be configured for differential ac susceptibility measurements. These sensors are compact, cheap, and can operate at room temperature. This opens opportunities for implementation of our differential magnetic assay in a portable, cheap, and ultrasensitive system for POC diagnostics. Volume amplification of the target with the RCA technique is furthermore an isothermal process and easy to execute, which is ideal for POC diagnostics. 
All of the steps of the assay can be implemented in a single, disposable microfluidic chip reactor, which requires only a small sample volume and reduces the risk of contamination $^{35}$. Several isothermal processes including the RCA have already been implemented on microfluidic chips, e.g., RCA on a microchip ${ }^{36}$, dendrimer-aptamer-RCA microfluidics for E. coli detection ${ }^{37}$, sensitive microRNA analysis using microfluidic exponential RCA ${ }^{38}$ and others ${ }^{39}$. The successful biofunctionalization of MNPs to antibodies, ligands, peptides nucleic acids, etc. makes them a versatile tool for many bioapplications ${ }^{40}$, especially diagnostics ${ }^{41}$. Our differential magnetic detection method is therefore, not limited to nucleic acids and can be used for detection of other target molecules.

By using a mixture of MNPs with significantly different hydrodynamic sizes and target-specific coatings, it has been shown that different DNA targets can be detected using volume amplified magnetic assays ${ }^{28,42}$. Such multiplexed magnetic assays, however, provide only qualitative results and have very low sensitivity to target analytes. They furthermore use turn-off based detection, which provides overlapping responses from the MNP mixture. The analysis of the detection response and, hence, the quantification of the targets is thus challenging. Our differential ac susceptibility measurement provides a starting zero signal, which makes signal analysis far more straightforward.

\section{Conclusion}

In conclusion, we have demonstrated a homogeneous differential magnetic assay for detection of DNA that can reach fM levels of sensitivity without additional amplification of the number of targets and washing steps. The removal of the background signal combined with fast binding kinetics offers opportunities for future applications of magnetic assays in rapid POC diagnostics.

\section{ASSOCIATED CONTENT}

Supporting information; Experimental method; Binding kinetics analysis for MNP hybridization to RCPs; Bimodal Cole-Cole relaxation fitting; Real components of differential ac susceptibility; Bimodal Cole-Cole relaxation fitting parameters; Performance of different sensors for detection of MNP labelled RCPs; Signal levels of measured imaginary ac susceptibility compared to the noise level of the SQUID gradiometer

\section{AUTHOR INFORMATION}

\section{Corresponding Author}

*E-mail: sobhan.sepehri@chalmers.se

\section{Author Contributions}

S.S., J.F.S, A.K., and D.W. conceived and designed the experiments. S.S. conducted the experiments and analysed the data. T.Z.G. contributed with the biochemical processes. A.J. helped with the fabrication of the microfluidics. J.B. performed reference ac susceptibility measurements and contributed in the discussion of the initial results. C.J contributed in the discussion of the results and analysis. S.S. and A.K. drafted the manuscript. All authors reviewed the manuscript and provided comments.

\section{Notes}

S.S., A.K., and D.W. are co-inventors of a patent filed on the subject of this work. The other authors declare no competing interests.

\section{Funding Sources}

This work was supported through the Swedish Foundation for Strategic Research (SSF) Grant "FLU-ID" No. SBE13-0125, the Swedish Research Council Grant 2015-03640, the Knut and Alice Wallenberg foundation, and the Swedish Infrastructure for Micro-and Nanofabrication-Myfab.

\section{ABBREVIATIONS}

POC, point of care; PCR, polymerase chain reaction; MNP, magnetic nanoparticle, PDMS, polydimethylsiloxane; SQUID, superconducting quantum interference device; RCP, rolling circle product; NC, negative control; RCA, rolling circle amplification.

\section{REFERENCES}

(1) Chen, S.; Shamsi, M. H. Biosensors-on-Chip: A Topical Review. J. Micromechanics Microengineering 2017, 27, 083001. https://doi.org/10.1088/1361-6439/aa7117.

(2) Kirsch, J.; Siltanen, C.; Zhou, Q.; Revzin, A.; Simonian, A. Biosensor Technology: Recent Advances in Threat Agent Detection and Medicine. Chem. Soc. Rev. 2013, 42 (22), 87338768. https://doi.org/10.1039/c3cs60141b.

(3) Yager, P.; Domingo, G. J.; Gerdes, J. Point-of-Care Diagnostics for Global Health. Annu. Rev. Biomed. Eng. 2008, 10 (1), 107144.

https://doi.org/10.1146/annurev.bioeng.10.061807.16052 4.

(4) Zhang, H.; Li, F.; Dever, B.; Li, X. F.; Le, X. C. DNA-Mediated Homogeneous Binding Assays for Nucleic Acids and Proteins. Chem. Rev. 2013, 113 (4), 2812-2841. https://doi.org/10.1021/cr300340p.

(5) Huang, X.; Liu, Y.; Yung, B.; Xiong, Y.; Chen, X. NanotechnologyEnhanced No-Wash Biosensors for in Vitro Diagnostics of Cancer. ACS Nano 2017, 11 (6), 5238-5292. https://doi.org/10.1021/acsnano.7b02618.

Chang, C.-C.; Chen, C.-C.; Wei, S.-C.; Lu, H.-H.; Liang, Y.-H.; Lin C.-W.; Chang, C.-C.; Chen, C.-C.; Wei, S.-C.; Lu, H.-H.; et al. Diagnostic Devices for Isothermal Nucleic Acid Amplification. Sensors 2012, 12 (6), 8319-8337. https://doi.org/10.3390/s120608319.

(7) Yang, S.; Rothman, R. E. PCR-Based Diagnostics for Infectious Diseases: Uses, Limitations, and Future Applications in AcuteCare Settings. Lancet Infect. Dis. 2004, 4 (6), 337-348. https://doi.org/10.1016/S1473-3099(04)01044-8.

(8) Su, H.; Meng, X.; Guo, Q.; Tan, Y.; Cai, Q.; Qin, H.; Meng, X. LabelFree DNAsensor with PCR-like Sensitivity Based on Background Reduction and Target-Triggered Polymerization Amplification. Biosens. Bioelectron. 2014, 52, 417-421. https://doi.org/10.1016/j.bios.2013.09.007.

Zhang, H.; Li, X. F.; Le, X. C. Binding-Induced DNA Assembly and Its Application to Yoctomole Detection of Proteins. Anal. $\begin{array}{llll}\text { Chem. 2012, } & 84 & \text { (2), 877-884. }\end{array}$ https://doi.org/10.1021/ac203207g.

(10) Chen, Y.-T.; Kolhatkar, A. G.; Zenasni, O.; Xu, S.; Lee, T. R.; Chen, Y.-T.; Kolhatkar, A. G.; Zenasni, O.; Xu, S.; Lee, T. R. Biosensing Using Magnetic Particle Detection Techniques. Sensors 2017, 17 (10), 2300-2336. https://doi.org/10.3390/s17102300.

(11) Tekin, H. C.; Gijs, M. A. M. Ultrasensitive Protein Detection: A Case for Microfluidic Magnetic Bead-Based Assays. Lab Chip 2013, 13 (24), 4711-4739. https://doi.org/10.1039/c3lc50477h.

(12) Lee, H.; Shin, T.-H.; Cheon, J.; Weissleder, R. Recent Developments in Magnetic Diagnostic Systems. Chem. Rev. 2015, 15 (19), 10690-10724. 
Reddy, L. H.; Arias, J. L.; Nicolas, J.; Couvreur, P. Magnetic Nanoparticles: Design and Characterization, Toxicity and Biocompatibility, Pharmaceutical and Biomedical Applications. Chem. Rev. 2012, 112 (11), 5818-5878. https://doi.org/10.1021/cr300068p.

14) Q. A. Pankhurst S. K. Jones, J. Dobson, J. C. Applications of Magnetic Nanoparticles in Biomedicine. J. Phys. D. Appl. Phys. 2003, 36, R167-R181. https://doi.org/10.1088/0022$3727 / 36 / 13 / 201$

(15) Astalan, A. P.; Ahrentorp, F.; Johansson, C.; Larsson, K.; Krozer A. Biomolecular Reactions Studied Using Changes in Brownian Rotation Dynamics of Magnetic Particles. Biosens. Bioelectron. 2004, $19 \quad$ (8), 945-951. https://doi.org/10.1016/j.bios.2003.09.005.

(16) Fougt Hansen, M.; Donolato, M.; Fock, J.; Strömberg, M.; Strømme, M.; Svedlindh, P. Sensor Systems with Magnetic and Optomagnetic Readout of Rolling Circle Amplification Products. In Rolling Circle Amplification (RCA): Toward New Clinical Diagnostics and Therapeutics; Demidov, V. V, Ed.; Springer International Publishing: Cham, 2016; pp 123-138. https://doi.org/10.1007/978-3-319-42226-8_11.

(17) Yang, C. C.; Yang, S. Y.; Chieh, J. J.; Horng, H. E.; Hong, C. Y.; Yang, H. C. Universal Behavior of Biomolecule-ConcentrationDependent Reduction in Ac Magnetic Susceptibility of Bioreagents. IEEE Magn. Lett. 2012, 3, 1500104. https://doi.org/10.1109/LMAG.2012.2183858.

(18) Dieckhoff, J.; Schrittwieser, S.; Schotter, J.; Remmer, H.; Schilling, M.; Ludwig, F. Single-Core Magnetic Markers in Rotating Magnetic Field Based Homogeneous Bioassays and the Law of Mass Action. J. Magn. Magn. Mater. 2015, 380, 205208. https://doi.org/10.1016/j.jmmm.2014.10.088.

(19) Ahrentorp, F.; Blomgren, J.; Jonasson, C.; Sarwe, A.; Sepehri, S. Eriksson, E.; Kalaboukhov, A.; Jesorka, A.; Winkler, D. Schneiderman, J. F.; et al. Sensitive Magnetic Biodetection Using Magnetic Multi-Core Nanoparticles and RCA Coils. J. Magn. Magn. Mater. 2017, 427 (September 2016), 14-18. https://doi.org/10.1016/j.jmmm.2016.10.041.

(20) Sepehri, S.; Eriksson, E.; Kalaboukhov, A.; Zardán Gómez de la Torre, T.; Kustanovich, K.; Jesorka, A.; Schneiderman, J. F.; Blomgren, I.; Johansson, C.; Strømme, M.; et al. VolumeAmplified Magnetic Bioassay Integrated with Microfluidic Sample Handling and High- Tc SQUID Magnetic Readout. APL $\begin{array}{llll}\text { Bioeng. 2018, } & 2 & \text { (1), } & 016102 .\end{array}$ https://doi.org/10.1063/1.4999713.

(21) Schrittwieser, S.; Pelaz, B.; Parak, W. J.; Lentijo-Mozo, S. Soulantica, K.; Dieckhoff, J.; Ludwig, F.; Guenther, A.; Tschöpe, A.; Schotter, J. Homogeneous Biosensing Based on Magnetic Particle Labels. Sensors (Switzerland) 2016, 16 (6). https://doi.org/10.3390/s16060828.

(22) Öisjöen, F.; Schneiderman, J. F.; Astalan, A. P.; Kalabukhov, A.; Johansson, C.; Winkler, D. The Need for Stable, MonoDispersed, and Biofunctional Magnetic Nanoparticles for One-Step Magnetic Immunoassays. J. Phys. Conf. Ser. 2010 200 (12), 122006. https://doi.org/10.1088/1742$6596 / 200 / 12 / 122006$.

(23) Tian, B.; Liao, X.; Svedlindh, P.; Strömberg, M.; Wetterskog, E. Ferromagnetic Resonance Biosensor for Homogeneous and Volumetric Detection of DNA. ACS Sensors 2018, 3, 10931101. https://doi.org/10.1021/acssensors.8b00048.

(24) Bejhed, R. S.; Østerberg, F. W.; Strömberg, M.; Svedlindh, P.; Mezger, A.; Donolato, M.; Hansen, M. F.; Rizzi, G.; Nilsson, M.; Strømme, M.; et al. On-Chip Detection of Rolling Circle Amplified DNA Molecules from Bacillus Globigii Spores and Vibrio Cholerae. Small 2014, 10 (14), 2877-2882. https://doi.org/10.1002/smll.201303325.

(25) Zardán Gómez De La Torre, T.; Ke, R.; Mezger, A.; Svedlindh, P.; Strømme, M.; Nilsson, M. Sensitive Detection of Spores Using Volume-Amplified Magnetic Nanobeads. Small 2012, 8 (14), 2174-2177. https://doi.org/10.1002/smll.201102632.

(26) Zardán Gómez de la Torre, T.; Mezger, A.; Herthnek, D.;
Johansson, C.; Svedlindh, P.; Nilsson, M.; Strømme, M.; Zardán Gómez de la Torre, T.; Mezger, A.; Herthnek, D.; et al. Detection of Rolling Circle Amplified DNA Molecules Using Probe-Tagged Magnetic Nanobeads in a Portable AC Susceptometer. Biosens. Bioelectron. 2011, 29 (1), 195-199. https://doi.org/10.1016/j.bios.2011.08.019.

(27) Bejhed, R. S.; Zardán Gómez de la Torre, T.; Donolato, M. Hansen, M. F.; Svedlindh, P.; Strömberg, M. Turn-on Optomagnetic Bacterial DNA Sequence Detection Using Volume-Amplified Magnetic Nanobeads. Biosens. Bioelectron. 2015, 66, 405-411. https://doi.org/10.1016/j.bios.2014.11.048.

(28) Strömberg, M.; Zardán Gómez de la Torre, T.; Nilsson, $\mathrm{M}_{\text {. }}$ Svedlindh, P.; Strømme, M. A Magnetic Nanobead-Based Bioassay Provides Sensitive Detection of Single- and Biplex Bacterial DNA Using a Portable AC Susceptometer. Biotechnol. J. 2014, 90 (1), 137-145. https://doi.org/10.1002/biot.201300348

(29) Blomgren, J.; Ahrentorp, F.; Ilver, D.; Jonasson, C.; Sepehri, S. Kalaboukhov, A.; Winkler, D.; Torre, T. Z. G. de la; Strømme, M.; Johansson, C.; et al. Development of a Sensitive InductionBased Magnetic Nanoparticle Biodetection Method. Nanomater. 2018, Vol. 8, Page 887 2018, 8 (11), 887. https://doi.org/10.3390/NAN08110887.

(30) Donolato, M.; Antunes, P.; Bejhed, R. S.; Zardán Gómez De La Torre, T.; Østerberg, F. W.; Strömberg, M.; Nilsson, M.; Strømme, M.; Svedlindh, P.; Hansen, M. F.; et al. Novel Readout Method for Molecular Diagnostic Assays Based on Optical Measurements of Magnetic Nanobead Dynamics. Anal. Chem 2015, $\quad 87 \quad$ (3), $\quad 1622-1629$ https://doi.org/10.1021/ac503191v.

(31) Tian, B.; Zardán Gómez De La Torre, T.; Donolato, M.; Hansen, M. F.; Svedlindh, P.; Strömberg, M. Multi-Scale Magnetic Nanoparticle Based Optomagnetic Bioassay for Sensitive DNA and Bacteria Detection. Anal. Methods 2016, 8 (25), 50095016. https://doi.org/10.1039/c6ay00721j.

(32) Ludwig, F.; Mäuselein, S.; Heim, E.; Schilling, M. Magnetorelaxometry of Magnetic Nanoparticles in Magnetically Unshielded Environment Utilizing a Differential Fluxgate Arrangement. Rev. Sci. Instrum. 2005, 76 (10), 1-3. https://doi.org/10.1063/1.2069776.

(33) Sun, X.; Feng, Z.; Zhi, S.; Lei, C.; Zhang, D.; Zhou, Y. An Integrated Microfluidic System Using a Micro-Fluxgate and Micro Spiral Coil for Magnetic Microbeads Trapping and Detecting. Sci. Rep. 2017, 7 (1), 12967 https://doi.org/10.1038/s41598-017-13389-x.

(34) Haraszczuk, R.; Yamada, S.; Kakikawa, M.; Ueno, T. Monitoring Minute Changes of Magnetic Markers' Susceptibility by SVGMR Needle-Type Probe. IEEE Trans. Magn. 2011, 47 (10), 2584-2587. https://doi.org/10.1109/TMAG.2011.2157959. Zanoli, L. M.; Spoto, G. Isothermal Amplification Methods for the Detection of Nucleic Acids in Microfluidic Devices. Biosensors 2013, 3 (1), 18-43. https://doi.org/10.3390/bios3010018.

(36) Sato, K.; Tachihara, A.; Renberg, B.; Mawatari, K.; Sato, K.; Tanaka, Y.; Jarvius, J.; Nilsson, M.; Kitamori, T. MicrobeadBased Rolling Circle Amplification in a Microchip for Sensitive DNA Detection. Lab Chip 2010, 10 (10), 1262-1266. https://doi.org/10.1039/b927460j.

(37) Jiang, Y.; Zou, S.; Cao, X. A Simple Dendrimer-Aptamer Based Microfluidic Platform for E. Coli 0157:H7 Detection and Signal Intensification by Rolling Circle Amplification. Sensors Actuators B Chem. 2017, 251, 976-984. https://doi.org/10.1016/J.SNB.2017.05.146.

(38) Cao, H.; Zhou, X.; Zeng, Y. Microfluidic Exponential Rolling Circle Amplification for Sensitive MicroRNA Detection Directly from Biological Samples. Sensors Actuators B Chem. 2019, 279, 447-457. https://doi.org/10.1016/J.SNB.2018.09.121.

(39) Giuffrida, M. C.; Spoto, G. Integration of Isothermal Amplification Methods in Microfluidic Devices: Recent 
Advances. Biosens. Bioelectron. 2017, 90 (November 2016), 174-186. https://doi.org/10.1016/j.bios.2016.11.045.

(40) Kudr, J.; Haddad, Y.; Richtera, L.; Heger, Z.; Cernak, M.; Adam, V.; Zitka, O. Magnetic Nanoparticles: From Design and Synthesis to Real World Applications. Nanomaterials 2017, 7 (9), 243. https://doi.org/10.3390/nano7090243.

(41) Haun, J. B.; Yoon, T.-J. J.; Lee, H.; Weissleder, R. Magnetic Nanoparticle Biosensors. Wiley Interdiscip. Rev. Nanomedicine Nanobiotechnology 2010, 2 (3), 291-304. https://doi.org/10.1002/wnan.84.

(42) Strömberg, M.; Zardán Gómez de la Torre, T.; Göransson, J.; Gunnarsson, K.; Nilsson, M.; Svedlindh, P.; Strømme, M. Multiplex Detection of DNA Sequences Using the VolumeAmplified Magnetic Nanobead Detection Assay. Anal. Chem. 2009

(9),

3398-3406.

https://doi.org/10.1021/ac900561r 